\title{
Early improvement of painful symptoms in MDD: is there an association in achieving remission?
}

Karl-Jürgen Bär

\author{
Address: Klinik für Psychiatrie Jena, Philosophenweg 3, 07743 Jena, Germany \\ from WPA Thematic Conference. Coercive Treatment in Psychiatry: A Comprehensive Review \\ Dresden, Germany. 6-8 June 2007 \\ Published: 19 December 2007 \\ BMC Psychiatry 2007, 7(Suppl I):S72 doi:10.1 I86/147I-244X-7-SI-S72
}

This abstract is available from: http://www.biomedcentral.com/I47I-244X/7/SI/S72

(c) 2007 Bär; licensee BioMed Central Ltd.

Previous research proposes strong interaction between chronic pain and depression. Depressed patients have been shown to be more vulnerable to pain complaints and, vice versa, chronic pain is frequently accompanied by depressive symptoms. The presentation will focus on treatment options of physical symptoms in depression. Some epidemiological data on physical symptoms in depression will be presented in the first part. The second part of the presentation will deal with some psychophysiological studies of pain in depression and will demonstrate the effect of depressive symptoms on pain perception. It will be demonstrated that painful stimuli are processed differentially depending on the localization of pain induction in depression. Suspected brain areas will be discussed in the course of the talk as well. The talk will furthermore present the current knowledge on treatment options for physical symptoms in depression. 